\title{
STATIONARY PARTITIONS AND PALM PROBABILITIES
}

\author{
GÜNTER LAST, ${ }^{*}$ Universität Karlsruhe
}

\begin{abstract}
A stationary partition based on a stationary point process $N$ in $\mathbb{R}^{d}$ is an $\mathbb{R}^{d}$-valued random field $\pi=\left\{\pi(x): x \in \mathbb{R}^{d}\right\}$ such that both $\pi(y) \in N$ for each $y \in \mathbb{R}^{d}$ and the random partition $\left\{\left\{y \in \mathbb{R}^{d}: \pi(y)=x\right\}: x \in N\right\}$ is stationary jointly with $N$. Stationary partitions may be considered as general versions of the stationary random tessellations studied in stochastic geometry. As in the special case of the Voronoi tessellation, a stationary partition can be used to relate the underlying stationary probability measure to the associated Palm probability measure of $N$. In doing so, we will develop some basic theory for stationary partitions and extend properties of stationary tessellations to our more general case. One basic idea is that the stationary measure is (up to a shift) a weighted version of the Palm measure, where the weight is the volume of the typical cell. We will make systematic use of a known modified probability measure. Finally, we use our approach to extend some recent results on the shift coupling of the stationary distribution and the Palm distribution.
\end{abstract}

Keywords: Point process; Palm probability; stationary partition; stationary tessellation; stochastic geometry; shift coupling

2000 Mathematics Subject Classification: Primary 60D05; 60G55

Secondary 60G60

\section{Introduction}

Let $N$ be a (simple) stationary point process in $\mathbb{R}^{d}$ defined on a probability space $(\Omega, \mathcal{A}, \mathrm{P})$. By a stationary partition (based on $N$ ) we mean an $\mathbb{R}^{d}$-valued (measurable) random field $\pi=\left\{\pi(x): x \in \mathbb{R}^{d}\right\}$ such that the field $\left\{\pi(x)-x: x \in \mathbb{R}^{d}\right\}$ is jointly stationary with $N$ and such that $\pi(x) \in N$ for all $x \in \mathbb{R}^{d}$, whenever $N \neq \varnothing$. A simple but important example is the Voronoi partition, where $\pi(x)$ is the point in $N$ closest to $x$ (chosen as the lexicographically smallest if there are several such closest points). In this case the random variables $\pi(x)$ are functions of $N$. In general, we do not assume anything about the joint distribution of $N$ and $\pi$, except joint stationarity. For $x \in N$, we refer to $C(x):=\left\{y \in \mathbb{R}^{d}: \pi(y)=x\right\}$ as the cell 'centred' at $x$. The system of cells is a (random) partition of $\mathbb{R}^{d}$ into Borel sets. We do not require that $x \in C(x)$, and for some of our results we can even allow some of the cells to be empty. Apart from requiring measurability of $\pi$ we will impose no further restrictions on the topological or geometrical structure of the cells. Stationarity implies that the statistical properties of $\{C(x): x \in N\}$ and the shifted partition $\{C(x)+z: x \in N\}$ are the same for all $z \in \mathbb{R}^{d}$. If the cells are all convex and compact and have nonempty interiors, then $\{C(x): x \in N\}$ is just a stationary tessellation as studied in stochastic geometry (see, e.g. [11] and [12]).

Received 26 August 2005; revision received 22 June 2006.

* Postal address: Institut für Stochastik, Universität Karlsruhe (TH), 76128 Karlsruhe, Germany.

Email address: last@math.uni-karlsruhe.de 
Our work has been largely motivated by two nice recent contributions to point process theory. Based on the Voronoi tessellation, Thorisson, in Chapter 9 of [14], provided an extensive and thorough discussion of the relationships between stationarity (as distributional invariance under all shifts) and point-stationarity (as distributional invariance under so-called bijective pointshifts). Holroyd and Peres [3] showed that an ergodic point process $N$ can be shifted to one of its points in such a way that the resulting process follows the Palm distribution of $N$. The remarkable fact is that this shift can be chosen to be a measurable function of $N$.

In Sections 4-8 we will extend many of the results of Chapter 9 of [14] from the Voronoi tessellation to general stationary partitions. In doing so, we will develop some basic theory for stationary partitions and generalize properties of stationary tessellation (see [11] and [12]) to our more general case. Moreover, we will use our approach to extend and complement some of the results of [3]. It is one of the aims of this paper to show that stationary partitions provide a good framework for accommodating all these results. Moreover, the refined Campbell theorem for stationary point processes will enable us to give very short proofs.

We will now give a more detailed description of the contents of the paper. Section 2 introduces stationary partitions in a purely deterministic framework. The probabilistic setting (taken from [8]) is established in Section 3. It allows us to treat stationary marked point processes or random fields that are stationary jointly with the point process $N$. Palm (probability) measures are introduced in Section 3. Section 4 shows how a stationary partition can be used to relate the stationary probability measure to the Palm probability measure. The basic idea is that the stationary measure is (up to a shift) a weighted version of the Palm measure, where the weight is the volume of the cell $C(0)$. Conversely, the Palm measure is (again up to a shift) a weighted version of the stationary measure, where the weight is the inverse of the volume of the cell, $V(0)$, containing the origin 0 . Although these are well-known and oft-investigated phenomena, [9] was perhaps the first paper that has rigorously introduced this change of measure in the context of marked point processes on the real line. We will derive these results from just one fundamental formula (see Theorem 4.1). It is a pleasing fact that this 'master formula' is a very simple consequence of the refined Campbell theorem. Section 5 contains a brief discussion of modified Palm probability measures as introduced in [5, p. 339] (see also [10] and [14]). While the Palm probability measure of $N$ can be interpreted as the conditional probability given that $N$ has a point at 0 (an event that actually has probability 0 ), its modified version describes the underlying stochastic experiment as seen from a randomly chosen point of $N$. Both concepts agree if and only if the sample intensity of $N$ is almost surely constant and, in particular, the underlying probability measure is ergodic. Section 6 provides conditional counterparts of the results in Section 4, and Section 7 deals with generalized stationary partitions in which only the members of some random set (jointly stationary with $N$ ) are allocated to the points of $N$. Section 8 shows how a stationary partition can be used to construct a stationary point process from a point-stationary one.

A stationary partition is balanced if each cell has the same volume. The spatial ergodic theorem then implies that this volume must be the inverse of the sample intensity of $N$ (see Proposition 9.1). It was proved in [3] that balanced stationary partitions do exist if $N$ is ergodic. By an easy generalization of the arguments in [3], we will show in Section 10 that this is also true without ergodicity. In fact, the stationary partition can be constructed to be a measurable function of $N$. Theorem 9.1 shows that, if $\pi$ is balanced, the shifted process $N-\pi(0)$ has the modified Palm distribution. For the Palm distribution, such an $N$-measurable shift coupling is possible if and only if the sample intensity is (almost surely) a constant (cf. Proposition 9.1 and Theorem 10.1). We finish the paper with some comments on shift couplings, in Section 11. 


\section{Stationary partitions}

In this paper all random elements are defined on a measurable space $(\Omega, \mathcal{A})$ equipped with a measurable flow $\theta_{x}: \Omega \rightarrow \Omega, x \in \mathbb{R}^{d}$. This is a family of measurable mappings such that $(\omega, x) \mapsto \theta_{x} \omega$ is measurable, $\theta_{0}$ is the identity on $\Omega$, and

$$
\theta_{x} \circ \theta_{y}=\theta_{x+y}, \quad x, y \in \mathbb{R}^{d},
$$

where, here and below, 'o' denotes composition.

We let $N$ denote the space of all locally finite subsets $\varphi \subset \mathbb{R}^{d}$ equipped with the $\sigma$-field $\mathcal{N}$ generated by the mappings $\varphi \mapsto \varphi(B):=\operatorname{card}(\varphi \cap B), B \in \mathcal{B}^{d}$. Here $\mathcal{B}^{d}$ denotes the Borel $\sigma$-field on $\mathbb{R}^{d}$. We will identify $\varphi \subset \mathbb{R}^{d}$ with the associated counting measure. A (simple) point process on $\mathbb{R}^{d}$ (see, e.g. [4]) is a measurable mapping $N: \Omega \rightarrow N$. It is said to be adapted to the flow, or stationary, if

$$
N(\omega, B+x)=N\left(\theta_{x} \omega, B\right), \quad \omega \in \Omega, x \in \mathbb{R}^{d}, B \in \mathcal{B}^{d},
$$

where we write $N(\omega, B):=N(\omega)(B)$, for $\omega \in \Omega$ and $B \in \mathcal{B}^{d}$. Throughout the paper $N$ will denote a flow-adapted point process. According to (2.2), we may think of $\theta_{x} \omega$ as $\omega$ shifted by the vector $-x$.

Example 2.1. Consider the measurable space $(\boldsymbol{N}, \mathcal{N})$ and define $\theta_{x} \varphi:=\varphi-x$ for $\varphi \in \boldsymbol{N}$ and $x \in \mathbb{R}^{d}$. Then $\left\{\theta_{x}: x \in \mathbb{R}^{d}\right\}$ is a measurable flow and the identity on $N$ is an adapted point process. If $\mathrm{P}$ is a stationary probability measure on $(\boldsymbol{N}, \mathcal{N})$, then we call the probability space $(\boldsymbol{N}, \mathcal{N}, \mathrm{P})$ a canonical space for stationary point processes.

A stationary partition (based on $N$ ) is a measurable mapping $\pi: \Omega \times \mathbb{R}^{d} \rightarrow \mathbb{R}^{d}$ that satisfies

$$
\pi(\omega, x) \in N(\omega), \quad \omega \in \Omega, N(\omega) \neq \varnothing,
$$

and is covariant, i.e.

$$
\pi\left(\theta_{y} \omega, x-y\right)=\pi(\omega, x)-y, \quad \omega \in \Omega, x, y \in \mathbb{R}^{d} .
$$

For convenience we also assume that $\pi(x)=x, x \in \mathbb{R}^{d}$, whenever $N=\varnothing$.

If $\pi$ is a stationary partition, then we define

$$
C^{\pi}(\omega, x)=\left\{y \in \mathbb{R}^{d}: \pi(\omega, y)=x\right\}, \quad \omega \in \Omega, x \in \mathbb{R}^{d} .
$$

Note that $C^{\pi}(\omega, x)=\varnothing$ whenever $x \notin N(\omega) \neq \varnothing$. The system $\left\{C^{\pi}(\omega, x): x \in N(\omega)\right\}$ forms a partition of $\mathbb{R}^{d}$ into measurable sets, provided that $N \neq \varnothing$. Although we do not make any topological or geometrical assumptions, we refer to $C^{\pi}(\omega, x)$ as the cell with (generalized) centre $x \in N(\omega)$. We do not assume that $x \in C^{\pi}(\omega, x)$, and some of the cells might be empty. A stationary tessellation as defined in stochastic geometry is then a stationary partition for which the cells are compact convex sets with nonempty interior (see [11] and [12]). Also, we write

$$
V^{\pi}(\omega, y):=\left\{z \in \mathbb{R}^{d}: \pi(\omega, z)=\pi(\omega, y)\right\}
$$

for the cell containing the site $y \in \mathbb{R}^{d}$, and note that

$$
V^{\pi}(\omega, y)=C^{\pi}(\omega, \pi(\omega, y)) .
$$


Whenever there is no risk of confusion we will omit the upper index, ' $\pi$ '. In accordance with the language of random variables, we will also most often suppress the explicit dependence on $\omega$. For instance, $C^{\pi}(x)$ then actually refers to the mapping $\omega \mapsto C^{\pi}(\omega, x)$.

The covariance property of a stationary partition $\pi$ implies that, for any $\omega \in \Omega$ and any $x \in \mathbb{R}^{d}$,

$$
\begin{aligned}
C\left(\theta_{y} \omega, 0\right) & =\left\{z: \pi\left(\theta_{y} \omega,(z+y)-y\right)=0\right\} \\
& =\{z: \pi(\omega, z+y)=y\} \\
& =C(\omega, y)-y
\end{aligned}
$$

and, more generally,

$$
C\left(\theta_{y} \omega, x\right)=C(\omega, x+y)-y, \quad \omega \in \Omega, x, y \in \mathbb{R}^{d} .
$$

Similarly,

$$
V\left(\theta_{x} \omega, 0\right)=\{y: \pi(\omega, y+x)=\pi(\omega, x)\}=V(\omega, x)-x .
$$

Example 2.2. For any $\varphi \in N \backslash\{\varnothing\}$ and any $x \in \mathbb{R}^{d}$, we let $R_{1}(\varphi, x), R_{2}(\varphi, x), \ldots$ be the points of $\varphi$ ordered according to increasing distance from $x$. Should some points of $\varphi$ be the same distance from $x$, we use the lexicographic order between them. If $\operatorname{card}(\varphi)=m<\infty$ then we set $R_{n}(\varphi, x) \equiv R_{m}(\varphi, x)$ for all $n \geq m$. For any $i \in \mathbb{N}$, the mapping

$$
(\omega, x) \mapsto R_{i}(N(\omega), x)
$$

is a stationary partition. For $i=1$, we call this the Voronoi partition based on $N$. In this case the closure of $\left\{y \in \mathbb{R}^{d}: R_{1}(N(\omega), y)=x\right\}$ is the Voronoi cell centred at $x \in N(\omega)$ (cf. [11] and [12]).

Remark 2.1. Let $\pi$ be a stationary partition based on a flow-adapted point process $N$. We wish to stress the fact that we do not consider $\left\{C^{\pi}(x): x \in N\right\}$ to be a random element in some suitable space of Borel partitions of $\mathbb{R}^{d}$. This is in contrast to the standard approach to stationary tessellations, taken in [11] and [12]. Consequently, if $\mathrm{Q}$ is some probability measure on $(\Omega, \mathcal{A})$ then we cannot speak of the distribution of $\left\{C^{\pi}(x): x \in N\right\}$. For similar reasons, we also cannot speak about the distributions of $V(0)$ and $C(0)$. The random variables of interest in this paper are the identity on $\Omega$, the mapping $\omega \mapsto \theta_{\pi(\omega, 0)}(\omega), \pi(0)$, and the volumes of the cells $V(0)$ and $C(0)$.

Although it is possible to introduce stationary tessellations as point processes on the space of closed sets, there are several reasons not to do so here. First, it would be an unnecessary restriction of generality. Second, we would like to avoid the technicalities that come with such an approach. Finally, we have no need to interpret the system of cells as a random element.

\section{The Palm measure}

A measure $\mathrm{P}$ on $(\Omega, \mathcal{A})$ is said to be stationary if it is invariant under the flow, i.e. if

$$
\mathrm{P} \circ \theta_{x}=\mathrm{P}, \quad x \in \mathbb{R}^{d},
$$

where $\theta_{x}$ is interpreted as a mapping from $\mathcal{A}$ to $\mathcal{A}$ in the usual way:

$$
\theta_{x} A:=\left\{\theta_{x} \omega: \omega \in A\right\}, \quad A \in \mathcal{A}, x \in \mathbb{R}^{d} .
$$


Throughout the paper $\mathrm{P}$ will denote a $\sigma$-finite stationary measure on $(\Omega, \mathcal{A})$. In the next sections we will most often assume that $\mathrm{P}$ is a probability measure. We work in the setting of [8]. Our use of an abstract flow $\left\{\theta_{x}: x \in \mathbb{R}^{d}\right\}$ makes the notation quite efficient and accommodates stationary marked point processes (see [1] and [5]) as well as stochastic processes (fields) jointly stationary with $N$ (see [14]).

Let $N$ be a point process adapted to the flow. The measure

$$
\mathrm{P}_{N}(A):=\iint \mathbf{1}\left(\theta_{x} \omega \in A, x \in[0,1]^{d}\right) N(\omega, \mathrm{d} x) \mathrm{P}(\mathrm{d} \omega), \quad A \in \mathcal{A},
$$

is called the Palm measure of $N$ (with respect to P); see [6]. It is $\sigma$-finite and satisfies the refined Campbell theorem,

$$
\iint f\left(\theta_{x} \omega, x\right) N(\omega, \mathrm{d} x) \mathrm{P}(\mathrm{d} \omega)=\iint f(\omega, x) \mathrm{d} x \mathrm{P}_{N}(\mathrm{~d} \omega),
$$

for all measurable functions $f: \Omega \times \mathbb{R}^{d} \rightarrow[0, \infty)$, where $\mathrm{d} x$ refers to integration with respect to the Lebesgue measure on $\mathbb{R}^{d}$. Using the standard convention mentioned in the previous section, we write this as

$$
\mathrm{E}\left[\int f\left(\theta_{x}, x\right) N(\mathrm{~d} x)\right]=\mathrm{E}_{\mathrm{P}_{N}}\left[\int f\left(\theta_{0}, x\right) \mathrm{d} x\right],
$$

where $\mathrm{E}$ and $\mathrm{E}_{\mathrm{P}_{N}}$ denote integration with respect to $\mathrm{P}$ and $\mathrm{P}_{N}$, respectively. The measure $\mathrm{P}_{N}$ is concentrated on the measurable set, $\Omega_{0}$, of all $\omega \in \Omega$ such that the origin 0 is in $N(\omega)$. The intensity of $N$ is the number

$$
\lambda_{N}:=\mathrm{E}\left[N\left([0,1]^{d}\right)\right]=\mathrm{P}_{N}(\Omega) .
$$

If this intensity is positive and finite, then the normalized Palm measure $\mathrm{P}_{N}^{0}:=\lambda_{N}^{-1} \mathrm{P}_{N}$ is called the Palm probability measure of $N$ (with respect to $\mathrm{P}$ ). Note that $\mathrm{P}_{N}$ and $\mathrm{P}_{N}^{0}$ are defined on the underlying space $(\Omega, \mathcal{A})$. The Palm distribution of $N, \mathrm{P}_{N}^{0}(N \in \cdot)$, is the distribution of $N$ under $\mathrm{P}_{N}^{0}$. It is concentrated on the measurable set, $N_{0}$, of all $\varphi \in N$ such that $0 \in \varphi$. The number $\mathrm{P}_{N}^{0}(A)$ can be interpreted as the conditional probability of $A \in \mathcal{A}$ given that $N$ has a point at the origin (see Proposition 9.3.4 of [5] or Theorem 11.6 of [4]).

\section{Basic properties of stationary partitions}

In this section we fix a stationary probability measure $\mathrm{P}$ on $(\Omega, \mathcal{A})$ and a stationary partition $\pi: \Omega \times \mathbb{R}^{d} \rightarrow \mathbb{R}^{d}$ based on a flow-adapted point process $N$. By (2.7) and (2.2), we have

$$
\left\{C\left(\theta_{y} \omega, x\right): x \in N\left(\theta_{y} \omega\right)\right\}=\{C(\omega, x)-y: x \in N(\omega)\}, \quad \omega \in \Omega, y \in \mathbb{R}^{d},
$$

so (as suggested by our terminology) the statistical properties of $\{C(x): x \in N\}$ are invariant under translations.

We will study the joint distribution of $\pi(0)$ and the $\Omega$-valued random variable $\theta_{\pi(0)}$, the latter defined by $\left(\theta_{\pi(0)}\right)(\omega):=\theta_{\pi(\omega, 0)} \omega$. Note that $N \circ \theta_{\pi(0)}=N-\pi(0)$ has a point at the origin whenever $N \neq \varnothing$. The following consequence of the refined Campbell theorem (and its conditional counterpart in Theorem 6.1) will be our main tool in this paper. 
Theorem 4.1. For all measurable functions $f, g: \Omega \rightarrow[0, \infty)$, we have

$$
\mathrm{E}\left[\mathbf{1}(N \neq \varnothing) f \cdot g\left(\theta_{\pi(0)}\right)\right]=\mathrm{E}_{\mathrm{P}_{N}}\left[g \cdot \int_{C(0)} f \circ \theta_{x} \mathrm{~d} x\right] .
$$

Proof. We follow the proof of Proposition 11.3 of [4], dealing with the special case of the Voronoi partition and $g \equiv 1$. The refined Campbell theorem (3.1) yields

$$
\mathrm{E}\left[\int h\left(\theta_{0}, x\right) N(\mathrm{~d} x)\right]=\mathrm{E}_{\mathrm{P}_{N}}\left[\int h\left(\theta_{x},-x\right) \mathrm{d} x\right]
$$

for all measurable $h: \Omega \times \mathbb{R}^{d} \rightarrow[0, \infty)$. We apply this formula with

$$
h(\omega, x):=f(\omega) g\left(\theta_{x} \omega\right) \mathbf{1}(x \in N(\omega), \pi(\omega, 0)=x) .
$$

The left-hand side of (4.2) then reduces to the left-hand side of (4.1). Furthermore,

$$
\begin{aligned}
h\left(\theta_{x} \omega,-x\right) & =f\left(\theta_{x} \omega\right) g\left(\theta_{-x}\left(\theta_{x} \omega\right)\right) \mathbf{1}\left(-x \in N\left(\theta_{x} \omega\right), \pi\left(\theta_{x} \omega, 0\right)=-x\right) \\
& =f\left(\theta_{x} \omega\right) g(\omega) \mathbf{1}(0 \in N(\omega), \pi(\omega, x)=0),
\end{aligned}
$$

where we have used the flow property (2.1) and the facts that $N$ is adapted to the basic flow (i.e. (2.2) holds) and $\pi$ is covariant. Hence, the right-hand sides of (4.2) and (4.1) coincide.

Taking $g \equiv 1$ in (4.1) yields the following inversion formula for Palm measures (see Satz 2.4 of [6]), adapted to our setting. For stationary tessellations the result can be found in Proposition 10.1 of [12].

Proposition 4.1. For all measurable functions $f: \Omega \rightarrow[0, \infty)$, we have

$$
\mathrm{E}[\mathbf{1}(N \neq \varnothing) f]=\mathrm{E}_{\mathrm{P}_{N}}\left[\int_{C(0)} f \circ \theta_{x} \mathrm{~d} x\right] .
$$

Taking $f \equiv 1$ in (4.4) yields

$$
\mathrm{E}_{\mathrm{P}_{N}}\left[|C(0)|_{d}\right]=\mathrm{P}(N \neq \varnothing),
$$

where $|B|_{d}$ denotes the Lebesgue measure of a Borel set $B \subset \mathbb{R}^{d}$. If $\mathrm{P}(N \neq \varnothing)=1$ and the intensity $\lambda_{N}$ of $N$ is finite, then

$$
\mathrm{E}_{\mathrm{P}_{N}^{0}}\left[|C(0)|_{d}\right]=\lambda_{N}^{-1}
$$

Our next proposition results from taking $f \equiv 1$ in (4.1). It shows that the distribution of $\theta_{\pi(0)}$ is absolutely continuous with respect to the Palm measure. As in the special case of the Voronoi partition, the density is the volume of the cell $C(0)$ (see Chapter 9 of [14]). This well-known phenomenon reflects the fact that the stationary measure is (up to a shift) a volume-biased version of the Palm measure.

Proposition 4.2. For all measurable functions $g: \Omega \rightarrow[0, \infty)$, we have

$$
\mathrm{E}_{\mathrm{P}_{N}}\left[|C(0)|_{d} \cdot g\right]=\mathrm{E}\left[\mathbf{1}(N \neq \varnothing) g\left(\theta_{\pi(0)}\right)\right] .
$$


Corollary 4.1. For any $\alpha \geq 0$, we have

$$
\mathrm{E}_{\mathrm{P}_{N}}\left[|C(0)|_{d}^{\alpha+1}\right]=\mathrm{E}\left[\mathbf{1}(N \neq \varnothing)|V(0)|_{d}^{\alpha}\right] .
$$

Proof. Equations (2.6) and (2.5) imply that, for all $\omega \in \Omega$,

$$
C\left(\theta_{\pi(\omega, 0)} \omega, 0\right)=C(\omega, \pi(\omega, 0))-\pi(\omega, 0)=V(\omega, 0)-\pi(\omega, 0) .
$$

Applying Proposition 4.2 with $g:=|C(0)|_{d}^{\alpha}$ then yields the result.

Remark 4.1. As their proofs rely only on the refined Campbell theorem, the preceding results also hold for $\sigma$-finite stationary measures on $(\Omega, \mathcal{A})$.

To prepare our next result we first note that (4.5) implies that

$$
\mathrm{P}_{N}\left(|C(0)|_{d}=\infty\right)=0 .
$$

We say that the stationary partition $\pi$ is P-proper if

$$
\mathrm{P}_{N}\left(|C(0)|_{d}=0\right)=0 .
$$

Equations (4.8) and (4.9) are equivalent to the fact that

$$
0<|C(\omega, x)|_{d}<\infty, \quad x \in N(\omega),
$$

holds for all $\omega$ outside an event of P-measure 0. Indeed, by (2.6) and the refined Campbell theorem, for any $B \in \mathscr{B}^{d}$ and any $c \in[0, \infty]$, we have

$$
|B|_{d} \mathrm{P}_{N}\left(|C(0)|_{d}=c\right)=\mathrm{E}\left[\int_{B} \mathbf{1}\left(|C(x)|_{d}=c\right) N(\mathrm{~d} x)\right] .
$$

As $B$ is arbitrary, the equality $\mathrm{P}_{N}\left(|C(0)|_{d}=c\right)=0$ is equivalent to the fact that the event $\left\{|C(x)|_{d} \neq c\right.$ for all $\left.x \in N\right\}$ has probability 1 .

The next result shows that, if $\pi$ is P-proper then the Palm measure is absolutely continuous with respect to the stationary distribution of $\theta_{\pi(0)}$. Again as in the special case of the Voronoi partition, the density is then the inverse of the volume of the cell $V(0)$ (see Chapter 9 of [14]). Hence, the Palm measure results from the stationary measure by volume debiasing and shifting $\pi(0)$ to 0 .

Proposition 4.3. If $\pi$ is $\mathrm{P}$-proper then, for all measurable functions $f: \Omega \rightarrow[0, \infty)$, we have

$$
\mathrm{E}_{\mathrm{P}_{N}}[f]=\mathrm{E}\left[\mathbf{1}(N \neq \varnothing)|V(0)|_{d}^{-1} \cdot f\left(\theta_{\pi(0)}\right)\right] .
$$

Proof. By (4.10) and Proposition 4.2, the event $A:=\left\{0<|C(0)|_{d}<\infty\right\}$ satisfies

$$
\mathrm{P}_{N}(\Omega \backslash A)=\mathrm{E}\left[\mathbf{1}(N \neq \varnothing) \mathbf{1}\left(\theta_{\pi(0)} \notin A\right)\right]=0 .
$$

We now apply Proposition 4.2 with $g:=\mathbf{1}_{A} \cdot f \cdot|C(0)|_{d}^{-1}$, to obtain

$$
\mathrm{E}_{\mathrm{P}_{N}}[f]=\int \mathbf{1}(N(\omega) \neq \varnothing)\left|C\left(\theta_{\pi(\omega, 0)} \omega, 0\right)\right|_{d}^{-1} \cdot f\left(\theta_{\pi(\omega, 0)} \omega\right) \mathrm{P}(\mathrm{d} \omega) .
$$

Using (4.7), the results follows. 
Corollary 4.2. If $\pi$ is $\mathrm{P}$-proper then (4.6) holds for any $\alpha \in \mathbb{R}$. In particular,

$$
\lambda_{N}=\mathrm{E}\left[\mathbf{1}(N \neq \varnothing)|V(0)|_{d}^{-1}\right] .
$$

Corollary 4.3. Assume both that $\mathrm{P}$ is a probability measure such that $\mathrm{P}(N=\varnothing)=0$ and $\lambda_{N}<\infty$, and that $\pi$ is $\mathrm{P}$-proper. Then

$$
\begin{aligned}
& \mathrm{E}_{\mathrm{P}_{N}^{0}}\left[|C(0)|_{d}\right]=\lambda_{N}^{-1}, \\
& \mathrm{E}_{\mathrm{P}_{N}^{0}}\left[|C(0)|_{d}\right] \leq \mathrm{E}\left[|V(0)|_{d}\right] .
\end{aligned}
$$

Proof. The first result follows from (4.6) with $\alpha=0$. The second is then a consequence of $\mathrm{E}\left[|V(0)|_{d}^{-1}\right] \geq \mathrm{E}\left[|V(0)|_{d}\right]^{-1}$ (Jensen's inequality) and (4.12).

We next consider the conditional distribution of $\pi(0)$ given $\theta_{\pi(0)}$. For the Voronoi case, we again refer the reader to [14].

Proposition 4.4. Assume that $\pi$ is P-proper. On the event $\{N \neq \varnothing\}$, the conditional distribution of $-\pi(0)$ given $\theta_{\pi(0)}$ is then the uniform distribution on $V(0)-\pi(0)$.

Proof. Note that $\{N=\varnothing\}$ is measurable with respect to $\theta_{\pi(0)}$. For simplicity, we may then assume that $\mathrm{P}(N=\varnothing)=0$. We consider measurable functions $g, h: \Omega \rightarrow[0, \infty)$. Applying Theorem 4.1 with $f:=h(-\pi(0))$ yields

$$
\mathrm{E}\left[g\left(\theta_{\pi(0)}\right) h(-\pi(0))\right]=\mathrm{E}_{\mathrm{P}_{N}}\left[g \cdot \int_{C(0)} h(x) \mathrm{d} x\right],
$$

where we have used the fact that $\pi\left(\theta_{x}, 0\right)=\pi\left(\theta_{0}, x\right)-x=-x$ on $C(0)$. Proposition 4.2 implies that

$$
\mathrm{E}_{\mathrm{P}_{N}}\left[g \cdot \int_{C(0)} h(x) \mathrm{d} x\right]=\mathrm{E}\left[g\left(\theta_{\pi(0)}\right)|V(0)|_{d}^{-1} \int_{C(0) \circ \theta_{\pi(0)}} h(x) \mathrm{d} x\right] .
$$

Since $C(0) \circ \theta_{\pi(0)}=V(0)-\pi(0)$, this completes the proof.

\section{The modified Palm probability measure}

Let $I \subset \mathcal{A}$ denote the invariant $\sigma$-field containing the sets $A \in \mathcal{A}$ with the property that $\theta_{x} A=A$ for all $x \in \mathbb{R}^{d}$. A function $f: \Omega \rightarrow \mathbb{R}$ is $\mathcal{I}$-measurable if and only if $f \circ \theta_{x}=f$ for all $x \in \mathbb{R}^{d}$. We let $\mathcal{I}^{\prime}$ denote the invariant $\sigma$-field on $N$, i.e. the system of all sets $C \in \mathcal{N}$ satisfying $C+x=C$ for all $x \in \mathbb{R}^{d}$.

Fixing a flow-adapted point process $N$, we define

$$
\mathcal{I}_{N}:=\left\{\{N \in C\}: C \in \mathcal{I}^{\prime}\right\} .
$$

Since $N$ is adapted, we have $I_{N} \subset \mathcal{L}$. Fixing a stationary probability measure $\mathrm{P}$ on $(\Omega, \mathcal{A})$, we next define

$$
\hat{N}:=\mathrm{E}\left[N\left([0,1]^{d}\right) \mid \mathcal{I}_{N}\right] .
$$

Stationarity implies that

$$
\mathrm{E}\left[N(B) \mid \mathcal{I}_{N}\right]=|B|_{d} \hat{N} \quad \text { P-a.s., } \quad B \in \mathcal{B}^{d} .
$$


We also note that $\{\hat{N}=0\}=\{N=\varnothing\}$ P-a.s. Here one inclusion is trivial while the other, $\{\hat{N}=0\} \subset\{N=\varnothing\}$, easily follows from (5.1). By spatial ergodic theory (see, e.g. Corollary 10.19 of [4]) we P-a.s. have

$$
\hat{N}=\lim _{n \rightarrow \infty} \frac{N\left(B_{n}\right)}{\left|B_{n}\right|_{d}}
$$

where $B_{n}$ is a ball of radius $n$ centred at the origin 0 . Therefore, $\hat{N}$ is called the sample intensity of $N$ (see [5] and [4]). The next lemma gives another property of $\hat{N}$.

Lemma 5.1. Assume that $\mathrm{P}(\hat{N}<\infty)=1$. Then

$$
\hat{N}=\mathrm{E}\left[N\left([0,1]^{d}\right) \mid \mathcal{I}\right] \quad \mathrm{P} \text {-a.s. }
$$

Proof. Let $\tilde{N}:=\mathrm{E}\left[N\left([0,1]^{d}\right) \mid \mathcal{I}\right]$. We first assume that $\lambda_{N}=\mathrm{E}[\hat{N}]<\infty$. Then the convergence (5.2) also holds in $L^{1}(\mathrm{P})$ (again see [4]). This fact, together with

$$
\mathrm{E}[N(B) \mid \mathcal{I}]=|B|_{d} \tilde{N} \quad \text { P-a.s., } \quad B \in \mathcal{B}^{d},
$$

implies that

$$
\mathrm{E}\left[\mathbf{1}_{A} \hat{N}\right]=\mathrm{E}\left[\mathbf{1}_{A} \tilde{N}\right], \quad A \in \mathcal{I},
$$

from which (5.3) follows. In the general case we define adapted point processes

$$
N_{m}:=\mathbf{1}(0<\hat{N} \leq m) N, \quad m \in \mathbb{N} .
$$

As $N_{m}$ has a finite intensity, we may apply the previous result to obtain

$$
\mathrm{E}\left[N_{m}\left([0,1]^{d}\right) \mid \mathcal{I}_{N_{m}}\right]=\mathbf{1}(0<\hat{N} \leq m) \tilde{N} \quad \text { P -a.s. }
$$

On the other hand we P-a.s. have $\{\hat{N}=0\}=\{N=\varnothing\}$ and, thus, $\left\{N_{m} \neq \varnothing\right\}=\{0<\hat{N} \leq m\}$ for $m \geq 1$. Therefore,

$$
\mathrm{E}\left[N_{m}\left([0,1]^{d}\right) \mid \mathcal{I}_{N_{m}}\right]=\mathrm{E}\left[N\left([0,1]^{d}\right) \mid \mathcal{I}_{N}\right] \quad \text { P-a.s. on }\{0<\hat{N} \leq m\} .
$$

Hence, (5.4) implies that (5.3) holds P-a.s. on the event $\{0<\hat{N}<\infty\}$. As $\tilde{N}=0$ and $N=\varnothing$ P-a.s. on $\{\hat{N}=0\}$, the assertion follows.

If $\mathrm{P}(0<\hat{N}<\infty)=1$ then we introduce the following modified version of the Palm probability measure:

$$
\mathrm{P}_{N}^{*}(A):=\mathrm{E}\left[\hat{N}^{-1} \int \mathbf{1}\left(\theta_{x} \in A, x \in[0,1]^{d}\right) N(\mathrm{~d} x)\right], \quad A \in \mathcal{A} .
$$

As $\hat{N}$ is invariant under the flow, we can use the refined Campbell theorem, (3.1), to rewrite this in differential form as

$$
\mathrm{dP}_{N}^{*}=\hat{N}^{-1} \mathrm{dP}_{N}
$$

The modified Palm probability measure was introduced in Section 9.1 of [5] and rediscovered in [10] and [14] (see also Chapter 11 of [4]). As explained in [5], $\mathrm{P}_{N}^{*}(A)$ can be interpreted as the conditional probability of $A$ given that 0 is a 'randomly chosen point' of $N$. Theorem 9.8.4 of [14] and our Theorems 9.1 and 10.1 will provide further support for this interpretation. 
Assume that $\mathrm{P}(0<\hat{N}<\infty)=1$. From the definition (5.5) and (3.1), we have the following modified refined Campbell theorem:

$$
\mathrm{E}\left[\hat{N}^{-1} \int f\left(\theta_{x}, x\right) N(\mathrm{~d} x)\right]=\mathrm{E}_{\mathrm{P}_{N}^{*}}\left[\int f\left(\theta_{0}, x\right) \mathrm{d} x\right] .
$$

Another, even more direct, consequence of definition (5.5) is that $\mathrm{P}_{N}^{*}$ coincides with $\mathrm{P}$ on $\mathcal{I}$ :

$$
\mathrm{P}_{N}^{*}(A)=\mathrm{P}(A), \quad A \in \mathcal{I} .
$$

Moreover, if $\mathrm{E}[\hat{N}]<\infty$ then $\mathrm{P}_{N}^{*}=\mathrm{P}_{N}^{0}$ if and only if

$$
\mathrm{P}(\hat{N}=\mathrm{E}[\hat{N}])=1 .
$$

If (5.8) is satisfied then $N$ is said to be pseudoergodic (see [10]). If $\mathrm{P}$ is ergodic, i.e. if $\mathrm{P}(A) \in\{0,1\}$ for all $A \in \mathcal{I}$, then $\mathrm{P}$ is also pseudoergodic. If $(\Omega, \mathcal{A})=(N, \mathcal{N}), \mathrm{P}$ is infinitely divisible, and $\lambda_{N}<\infty$, then (5.8) is actually equivalent to ergodicity of $\mathrm{P}$ (cf. Proposition 6.4.10 of [5]). In general, however, (5.8) is clearly a weaker condition than ergodicity. This is shown, for instance, by the following version of an example in Section 6.1 of [5]. Other examples can be found in [10].

Example 5.1. Assume that $(\Omega, \mathcal{A})=(\boldsymbol{N}, \mathcal{N})$. For any $c>0$, we let $\mathrm{P}_{c}$ be the distribution of a stationary Poisson process of intensity $c$. Let $y \in \mathbb{R}^{d}$ and let $\mathrm{Q}$ be the distribution of $N \cup(N+y)$ under $\mathrm{P}_{1}$. Under both $\mathrm{P}_{2}$ and $\mathrm{Q}$ we have $\hat{N}=2$ almost surely. Therefore, the stationary probability measure

$$
\mathrm{P}:=p \mathrm{P}_{2}+(1-p) \mathrm{Q}, \quad 0<p<1,
$$

is pseudoergodic. This measure is not ergodic (cf. Section 6.2.6 of [5]).

The ergodic decomposition theorem (see, e.g. Proposition 10.24 and Theorem 10.26 of [4]) shows that $N$ is pseudoergodic if and only if $\mathrm{P}$ is a mixture of ergodic probability measures with respect to almost all of which $N$ has the same intensity.

Finally, we mention another important invariance property of measures on $(\Omega, \mathcal{A})$. A covariant mapping $\pi: \Omega \times \mathbb{R}^{d} \rightarrow \mathbb{R}^{d}$ is called a bijective point map (see [14]) if

$$
\pi(\omega, x) \in N(\omega)
$$

whenever $x \in N(\omega)$ and the restriction of $\pi(\omega, \cdot)$ on $N(\omega)$ is bijective. Following [14] and [2], we say that a measure $\mathrm{Q}$ on $(\Omega, \mathcal{A})$ is point-stationary (with respect to $N)$ if $\mathrm{Q}(0 \notin N)=0$ and

$$
\mathrm{Q}(\cdot)=\mathrm{Q}\left(\theta_{\pi(0)} \in \cdot\right)
$$

holds for all bijective point maps $\pi: \Omega \rightarrow \mathbb{R}^{d}$ such that $\pi(0)$ is $\sigma(N)$-measurable. Here $\theta_{\pi}$ denotes the mapping $\omega \mapsto \theta_{\pi(\omega, 0)} \omega$. By Satz 4.3 of [7], the Palm measure $\mathrm{P}_{N}$ is pointstationary. In this case (5.9) actually holds for all bijective point maps. The proof of Theorem 3.1 of [2] shows that this also true for the modified Palm probability $\mathrm{P}_{N}^{*}$. One has to use the modified refined Campbell theorem, (5.6). Conversely, it was shown in Theorem 4.1 of [2] that any $\sigma$-finite, point-stationary measure is the Palm measure of a $\sigma$-finite, stationary measure on $(\Omega, \mathcal{A})$. 


\section{Conditional expectations}

In this section we consider a stationary probability measure $\mathrm{P}$ on $(\Omega, \mathcal{A})$ and a stationary partition $\pi$ based on a flow-adapted point process $N$ such that $\mathrm{P}(0<\hat{N}<\infty)=1$. In particular, $\mathrm{P}(N=\varnothing)=0$. First we provide a conditional version of Theorem 4.1.

Theorem 6.1. For all measurable functions $f, g: \Omega \rightarrow[0, \infty)$, we have

$$
\mathrm{E}\left[f \cdot g\left(\theta_{\pi(0)}\right) \mid \mathcal{I}\right]=\hat{N} \mathrm{E}_{\mathrm{P}_{N}^{*}}\left[g \cdot \int_{C(0)} f \circ \theta_{x} \mathrm{~d} x \mid \mathcal{I}\right] \quad \mathrm{P} \text {-a.s. },
$$

for any choice of the conditional expectations considered here.

Proof. We fix $f$ and $g$ as well as two versions of the involved conditional expectations. In view of (5.7), we have to show that

$$
\mathrm{E}\left[\hat{N}^{-1} \tilde{g} f g\left(\theta_{\pi(0)}\right)\right]=\mathrm{E}_{\mathrm{P}_{N}^{*}}\left[\tilde{g} g \int_{C(0)} f \circ \theta_{x} \mathrm{~d} x\right]
$$

for any $\mathcal{L}$-measurable function $\tilde{g}: \Omega \rightarrow[0, \infty)$. However, this follows from the modified refined Campbell theorem, (5.6), in the same way that (4.1) was derived from (3.1): all we have to do is replace $g$ by the product of $g$ and $\tilde{g}$.

As in the previous section, we may draw several corollaries. The Voronoi case has been treated in Chapter 9 of [14].

Proposition 6.1. For all measurable functions $f: \Omega \rightarrow[0, \infty)$, we have

$$
\mathrm{E}[f \mid \tau]=\hat{N} \mathrm{E}_{\mathrm{P}_{N}^{*}}\left[\int_{C(0)} f \circ \theta_{x} \mathrm{~d} x \mid \tau\right] \quad \mathrm{P} \text {-a.s. }
$$

Proposition 6.2. Let $g: \Omega \rightarrow[0, \infty)$ be measurable. Then

$$
\mathrm{E}_{\mathrm{P}_{N}^{*}}\left[|C(0)|_{d} \cdot g \mid \mathcal{I}\right]=\hat{N}^{-1} \mathrm{E}\left[g\left(\theta_{\pi(0)}\right) \mid \mathcal{I}\right] \quad \mathrm{P} \text {-a.s. }
$$

Also, if $\pi$ is $\mathrm{P}$-proper then

$$
\mathrm{E}_{\mathrm{P}_{N}^{*}}[g \mid \mathcal{I}]=\hat{N}^{-1} \mathrm{E}\left[|V(0)|_{d}^{-1} \cdot g\left(\theta_{\pi(0)}\right) \mid \tau\right] \quad \mathrm{P} \text {-a.s. }
$$

Corollary 6.1. For any $\alpha \geq 0$,

$$
\mathrm{E}_{\mathrm{P}_{N}^{*}}\left[|C(0)|_{d}^{\alpha+1} \mid \mathcal{I}\right]=\hat{N}^{-1} \mathrm{E}\left[|V(0)|_{d}^{\alpha} \mid \mathcal{I}\right] \quad \mathrm{P} \text {-a.s. }
$$

If $\pi$ is $\mathrm{P}$-proper, then this is true for all $\alpha \in \mathbb{R}$.

Assume that $\pi$ is P-proper. Letting $\alpha=-1$ in (6.3) gives

$$
\hat{N}=\mathrm{E}\left[|V(0)|_{d}^{-1} \mid \mathcal{I}\right] \quad \mathrm{P} \text {-a.s. }
$$

and (6.2) implies that

$$
\mathrm{E}_{\mathrm{P}_{N}^{*}}[g]=\mathrm{E}\left[|V(0)|_{d}^{-1} \cdot \mathrm{E}\left[|V(0)|_{d}^{-1} \mid \tau\right]^{-1} \cdot g\left(\theta_{\pi(0)}\right)\right] .
$$




\section{Extended stationary partitions}

Let $N$ denote a flow-adapted point process. An extended stationary partition (based on $N$ ) is a pair $(Z, \pi)$ consisting of a measurable set $Z: \Omega \rightarrow \mathcal{B}^{d}$ and a mapping $\pi: \Omega \times \mathbb{R}^{d} \rightarrow \mathbb{R}^{d}$ that are both covariant and are such that $\pi(x) \in N$ whenever $x \in Z$ and $\{Z=\varnothing\}=\{N=\varnothing\}$. Measurability of $Z$ just means that $(\omega, x) \mapsto \mathbf{1}(x \in Z(\omega))$ is measurable, while covariance of $Z$ means that

$$
Z\left(\theta_{x} \omega\right)=Z(\omega)-x, \quad \omega \in \Omega, x \in \mathbb{R}^{d} .
$$

Covariance of $\pi$ is defined in (2.3). If $(Z, \pi)$ is an extended stationary partition, we define

$$
C(\omega, x):=\{y \in Z(\omega): \pi(\omega, y)=x\}, \quad \omega \in \Omega, x \in \mathbb{R}^{d},
$$

as in (2.4). Theorems 4.1 and 6.1 can be generalized as follows.

Theorem 7.1. Let $\mathrm{P}$ be a stationary probability measure on $(\Omega, \mathcal{A})$. Let $(Z, \pi)$ be an extended stationary partition and let $f, g: \Omega \rightarrow[0, \infty)$ be measurable functions. Then we have

$$
\mathrm{E}\left[\mathbf{1}(0 \in Z) f \cdot g\left(\theta_{\pi(0)}\right)\right]=\mathrm{E}_{\mathrm{P}_{N}}\left[g \cdot \int_{C(0)} f \circ \theta_{x} \mathrm{~d} x\right],
$$

where $C(0)$ is as defined in (7.1). If $\mathrm{P}(0<\hat{N}<\infty)=1$ then

$$
\mathrm{E}\left[\mathbf{1}(0 \in Z) f \cdot g\left(\theta_{\pi(0)}\right) \mid \tau\right]=\hat{N} \mathrm{E}_{\mathrm{P}_{N}^{*}}\left[g \cdot \int_{C(0)} f \circ \theta_{x} \mathrm{~d} x \mid \tau\right] \quad \mathrm{P} \text {-a.s. }
$$

for any choice of the conditional expectations considered here.

Proof. In proving (7.2) we follow the proof of Theorem 4.1. We just have to multiply the function $h$, defined in (4.3), by $\mathbf{1}(0 \in Z(\omega))$. The argument for (7.3) is similar.

\section{From Palm probability measures to stationary probability measures}

We fix a flow-adapted point process $N$ and a probability measure $\mathrm{Q}$ on $(\Omega, \mathcal{A})$ such that $\mathrm{Q}(N=\varnothing)=0$. However, we do not assume that $\mathrm{Q}$ is stationary. We also fix a stationary partition $\pi$ based on $N$ and assume that (4.10) holds for all $\omega$ outside an event of Q-measure 0 .

Motivated by Proposition 4.3 , we define another measure, $\mathrm{Q}^{0}$, on $(\Omega, \mathcal{A})$ with density $|V(0)|_{d}^{-1}$ with respect to $\mathrm{Q}$. This means that

$$
\mathrm{E}_{\mathrm{Q}^{0}}[f]=\mathrm{E}_{\mathrm{Q}}\left[|V(0)|_{d}^{-1} \cdot f\right]
$$

for all measurable functions $f: \Omega \rightarrow[0, \infty)$. Since we have assumed (4.10) to hold for Q-almost every $\omega$, we have

$$
\mathrm{Q}\left(|V(0)|_{d}=0\right)+\mathrm{Q}\left(|V(0)|_{d}=\infty\right)=0 .
$$

Hence, $\mathrm{Q}^{0}$ is $\sigma$-finite. It turns out that stationarity of $\mathrm{Q}$ is equivalent to point-stationarity of $\mathrm{Q}^{0}\left(\theta_{\pi(0)} \in \cdot\right)$ and conditional uniformity of $-\pi(0)$ given $\theta_{\pi(0)}$ (see the end of Section 5 for a brief discussion of point-stationarity). This fact is again a generalization of a result on Voronoi tessellations in Chapter 9 of [14] to our much more general case.

Theorem 8.1. The measure $\mathrm{Q}$ is stationary if and only if the measure $\mathrm{Q}^{0}\left(\theta_{\pi(0)} \in \cdot\right)$ is pointstationary and the conditional distribution $\mathrm{Q}\left(-\pi(0) \in \cdot \mid \theta_{\pi(0)}\right)$ can be chosen to be the uniform distribution on $V(0)-\pi(0)$. 
Proof. We have assumed that $\mathrm{Q}(N=\varnothing)=0$ and that $\pi$ is Q-proper. If $\mathrm{Q}$ is stationary then Proposition 4.3 shows that $\mathrm{Q}^{0}\left(\theta_{\pi(0)} \in \cdot\right)$ is the Palm measure of $\mathrm{Q}$. As mentioned at the end of Section 5, a Palm measure is point-stationary. Proposition 4.4 implies the asserted conditional uniformity.

Now we assume the point-stationarity of $\mathrm{Q}^{0}\left(\theta_{\pi(0)} \in \cdot\right)$ and the conditional uniformity of $-\pi(0)$. Owing to the main result of [2], there is a unique $\sigma$-finite, stationary measure $\mathrm{P}$ on $(\Omega, \mathcal{A})$ such that $\mathrm{P}(N=\varnothing)=0$ and $\mathrm{Q}^{0}\left(\theta_{\pi(0)} \in \cdot\right)=\mathrm{P}_{N}$. Our goal is to show that $\mathrm{Q}=\mathrm{P}$. Applying first Proposition 4.2 (cf. Remark 4.1) and then the definition of $\mathrm{Q}^{0}$ yields, for all measurable functions $g: \Omega \rightarrow[0, \infty)$,

$$
\begin{aligned}
\mathrm{E}\left[g\left(\theta_{\pi(0)}\right)\right] & =\mathrm{E}_{\mathrm{P}_{N}}\left[g \cdot|C(0)|_{d}\right] \\
& =\mathrm{E}_{\mathrm{Q}}\left[|V(0)|_{d}^{-1} \cdot g\left(\theta_{\pi(0)}\right) \cdot\left|C(0) \circ \theta_{\pi(0)}\right|_{d}\right] \\
& =\mathrm{E}_{\mathrm{Q}}\left[g\left(\theta_{\pi(0)}\right)\right],
\end{aligned}
$$

where we have used the fact that $C(0) \circ \theta_{\pi(0)}=V(0)-\pi(0)$. Hence, $\mathrm{P}$ is a probability measure and

$$
\mathrm{P}\left(\theta_{\pi(0)} \in \cdot\right)=\mathrm{Q}\left(\theta_{\pi(0)} \in \cdot\right) .
$$

By Proposition 4.4 and the assumptions, we then have

$$
\mathrm{P}\left(\pi(0) \in \cdot \mid \theta_{\pi(0)}\right)=\mathrm{Q}\left(\pi(0) \in \cdot \mid \theta_{\pi(0)}\right) \quad \mathrm{P} \text {-a.s. }
$$

Since $\omega=\theta_{-\pi(\omega, 0)}\left(\theta_{\pi(\omega, 0)} \omega\right)$ for all $\omega \in \Omega,(8.2)$ and (8.3) give $\mathrm{P}=\mathrm{Q}$.

We now assume that $\mathrm{Q}$ is a probability measure such that $\mathrm{Q}(0<\eta<\infty)=1$, where

$$
\eta:=\mathrm{E}_{\mathrm{Q}}\left[|V(0)|_{d}^{-1} \mid \tau\right] .
$$

Motivated by (6.5), we define another probability measure, $\mathrm{Q}^{*}$, on $(\Omega, \mathcal{A})$ that has density $\eta^{-1} \cdot|V(0)|_{d}^{-1}$ with respect to Q. This means that

$$
\mathrm{E}_{\mathrm{Q}^{*}}[f]=\mathrm{E}_{\mathrm{Q}}\left[\eta^{-1} \cdot|V(0)|_{d}^{-1} \cdot f\right]
$$

for all measurable functions $f: \Omega \rightarrow[0, \infty)$.

Theorem 8.2. The measure $Q$ is stationary if and only if the measure $Q^{*}\left(\theta_{\pi(0)} \in \cdot\right)$ is pointstationary and the conditional distribution $\mathrm{Q}\left(-\pi(0) \in \cdot \mid \theta_{\pi(0)}\right)$ can be chosen to be the uniform distribution on $V(0)-\pi(0)$.

Proof. Again we have to prove only one implication. Let us assume that $\mathrm{Q}^{*}\left(\theta_{\pi(0)} \in \cdot\right)$ is point-stationary and let $\mathrm{P}$ be a $\sigma$-finite, stationary measure on $(\Omega, \mathcal{A})$ such that $\mathrm{P}(N=\varnothing)=0$ and $\mathrm{Q}^{*}\left(\theta_{\pi(0)} \in \cdot\right)=\mathrm{P}_{N}$. As in (8.1), we obtain

$$
\mathrm{E}\left[g\left(\theta_{\pi(0)}\right)\right]=\mathrm{E}_{\mathrm{Q}}\left[\eta^{-1} g\left(\theta_{\pi(0)}\right)\right] .
$$

This shows, in particular, that $\mathrm{P}(\eta=0)+\mathrm{P}(\eta=\infty)=0$. Since $\eta$ is $\mathcal{I}$-measurable, we have

$$
\eta=\eta \circ \theta_{\pi(0)}
$$

In particular, $\eta$ is $\sigma\left(\theta_{\pi(0)}\right)$-measurable. By (8.5) we can rewrite (8.4) as

$$
\mathrm{E}_{\hat{\mathrm{P}}}\left[g\left(\theta_{\pi(0)}\right)\right]=\mathrm{E}_{\mathrm{Q}}\left[g\left(\theta_{\pi(0)}\right)\right],
$$


where $\hat{\mathrm{P}}$ is the measure with density $\eta$ with respect to $\mathrm{P}$. This is a stationary probability measure. Since $\eta$ is $\sigma\left(\theta_{\pi(0)}\right)$-measurable it follows that

$$
\hat{\mathrm{P}}\left(\pi(0) \in \cdot \mid \theta_{\pi(0)}\right)=\mathrm{P}\left(\pi(0) \in \cdot \mid \theta_{\pi(0)}\right) \quad \hat{\mathrm{P}} \text {-a.s. }
$$

As in the previous proof, this yields $\hat{\mathrm{P}}=\mathrm{Q}$ and, hence, the stationarity of $\mathrm{Q}$.

\section{Balanced stationary partitions}

We consider a stationary probability measure $\mathrm{P}$ on $(\Omega, \mathcal{A})$ and a flow-adapted point process $N$ such that $\mathrm{P}(0<\hat{N}<\infty)=1$. Essentially following [3], we call a stationary partition $\pi$ (based on $N$ ) balanced (with respect to $\mathrm{P}$ ) if

$$
\mathrm{P}\left(|C(x)|_{d}=\hat{N}^{-1} \text { for all } x \in N\right)=1 .
$$

As at (4.11), it follows that a stationary partition $\pi$ is balanced if and only if

$$
\mathrm{P}_{N}^{*}\left(|C(0)|_{d}=\hat{N}^{-1}\right)=1 .
$$

The next result explains the occurrence of the sample intensity $\hat{N}$ in (9.1).

Proposition 9.1. Let $\xi$ be a random variable such that $\mathrm{P}(0<\xi<\infty)=1$ and let $\pi$ be a stationary partition based on N. Assume that

$$
\mathrm{P}\left(|C(x)|_{d}=\xi \text { for all } x \in N\right)=1 \text {. }
$$

Then $\mathrm{P}\left(\xi=\hat{N}^{-1}\right)=1$.

Proof. The random measure

$$
M(B):=\int \mathbf{1}(\pi(x) \in B) \mathrm{d} x, \quad B \in \mathscr{B}^{d},
$$

is adapted to the flow (i.e. $M \circ \theta_{x}(B)=M(B+x)$ ) and, hence, stationary. Corollary 10.19 of [4] implies the almost-sure existence of the $\mathcal{L}$-measurable limit

$$
\hat{M}:=\lim _{n \rightarrow \infty} \frac{M\left(B_{n}\right)}{\left|B_{n}\right|_{d}},
$$

as in (5.2). On the other hand, from (9.2) we have $M(B)=\xi N(B)$, so (5.2) implies that $\hat{M}=\xi \hat{N}$. Hence, $\xi$ is almost surely $\tau$-measurable and (6.4) implies that $\xi^{-1}=\hat{N}$.

We say that a stationary partition $\pi$ is $N$-measurable, if $\pi(0)$ is $\sigma(N)$-measurable. It has been proved in [3] that balanced, $N$-measurable stationary partitions exist if $\mathrm{P}$ is ergodic. Theorem 10.1, below, shows that this is true also without ergodicity.

Remark 9.1. Assume that $\lambda_{N}=\mathrm{E}[\hat{N}]<\infty$. The authors of [3] said that a stationary partition $\pi$ is balanced if

$$
\mathrm{P}\left(|C(x)|_{d}=\lambda_{N}^{-1} \text { for all } x \in N\right)=1 .
$$

Proposition 9.1 shows that (9.3) can only hold if $N$ is pseudoergodic (see Section 5). 
The significance of balanced, stationary partitions is due to the following theorem. The result extends Theorem 13 of [3] from the pseudoergodic case to the general case.

Theorem 9.1. Let $\pi$ be a stationary partition. Then $\pi$ is balanced if and only if

$$
\mathrm{P}\left(\theta_{\pi(0)} \in \cdot\right)=\mathrm{P}_{N}^{*} \cdot
$$

Proof. Let $g: \Omega \rightarrow[0, \infty)$ be a measurable function. If $\pi$ is balanced then from Proposition 6.2 we obtain

and, in particular, (9.4).

$$
\mathrm{E}_{\mathrm{P}_{N}^{*}}[g \mid \mathcal{I}]=\mathrm{E}\left[g\left(\theta_{\pi(0)}\right) \mid \mathcal{I}\right] \quad \mathrm{P} \text {-a.s. }
$$

Let us now assume that (9.4) holds. Choosing $g \equiv 1$ in (6.1) yields

$$
\mathrm{E}_{\mathrm{P}_{N}^{*}}\left[\hat{N} \cdot|C(0)|_{d}\right]=1 \text {. }
$$

Choosing $g \equiv \hat{N} \cdot|C(0)|_{d}$ yields

$$
\mathrm{E}_{\mathrm{P}_{N}^{*}}\left[\hat{N}^{2} \cdot|C(0)|_{d}^{2}\right]=\mathrm{E}_{\mathrm{P}_{N}^{*}}\left[\hat{N} \cdot|C(0)|_{d}\right]=1 .
$$

Since this implies (9.1), $\pi$ is balanced.

\section{Existence of balanced stationary partitions}

The existence of balanced stationary partitions is a consequence of the following lemma.

Lemma 10.1. For any $\lambda>0$, there exist covariant mappings $A_{\lambda}$, from $N$ into the system of Borel subsets of $\mathbb{R}^{d}$, and $\pi_{\lambda}: N \times \mathbb{R}^{d} \rightarrow \mathbb{R}^{d}$ such that

$$
(\lambda, \varphi, y) \mapsto\left(\mathbf{1}\left(y \in A_{\lambda}(\varphi)\right), \pi_{\lambda}(\varphi, y)\right)
$$

is a measurable mapping and, for any $\lambda>0$, the sets

$$
C_{\lambda}(\varphi, x):=\left\{y \in A_{\lambda}(\varphi): \pi_{\lambda}(\varphi, y)=x\right\}, \quad \varphi \in N, x \in \mathbb{R}^{d},
$$

satisfy

$$
0<\left|C_{\lambda}(\varphi, x)\right|_{d} \leq \lambda^{-1}, \quad \varphi \in N, x \in \varphi,
$$

and the mapping $T_{\lambda}: N \rightarrow N$ defined by

$$
T_{\lambda}(\varphi):=\left\{x \in \varphi:\left|C_{\lambda}(\varphi, x)\right|_{d}<\lambda^{-1}\right\}
$$

satisfies

$$
\left\{\varphi: T_{\lambda}(\varphi) \neq \varnothing\right\} \cap\left\{\varphi: A_{\lambda}(\varphi) \neq \mathbb{R}^{d}\right\}=\varnothing .
$$

An explicit example of the mappings in the preceding lemma is provided by the continuous version of the Gale-Shapley allocation algorithm of [3]. It is not difficult to come up with modifications of this example.

Theorem 10.1. Let $\mathrm{P}$ be a stationary probability measure and $N$ a flow-adapted point process such that $\mathrm{P}(0<\hat{N}<\infty)=1$. Let the mappings $A_{\lambda}$ and $\pi_{\lambda}, \lambda>0$, be as defined in Lemma 10.1. Then the mapping $\delta: \Omega \times \mathbb{R}^{d} \rightarrow \mathbb{R}^{d}$ defined by

$$
\delta(\omega, x):= \begin{cases}\pi_{\hat{N}(\omega)}(N(\omega), x) & \text { if } 0<\hat{N}(\omega)<\infty \text { and } x \in A_{\hat{N}(\omega)}(N(\omega)), \\ R_{1}(N(\omega), x) & \text { otherwise, }\end{cases}
$$

is an $N$-measurable stationary partition that is balanced with respect to $\mathrm{P}$. Here $R_{1}(N(\omega), x)$ is as defined in Example 2.2. 
Proof. In the ergodic case the result can be proved along the same lines as Theorem 1 of [3]. In the general case we could use ergodic decomposition. For the reader's convenience we give a direct argument.

Assuming without loss of generality that $0<\hat{N}<\infty$ holds identically on $\Omega$, we define $Z:=A_{\hat{N}}(N)$ and

$$
\pi(\omega, x):=\pi_{\hat{N}(\omega)}(N(\omega), x) \quad \text { for } x \in Z(\omega) .
$$

For $x \notin Z(\omega)$, we define $\pi(\omega, x):=R_{1}(N(\omega, x))$ if $N(\omega) \neq \varnothing$. The pair $(Z, \pi)$ is an extended stationary partition in the sense of Section 7. By (7.3) we P-a.s. have

$$
\mathrm{P}(0 \in Z \mid \mathcal{I})=\mathrm{E}_{\mathrm{P}_{N}^{*}}\left[\hat{N} \cdot|C(0)|_{d} \mid \mathcal{I}\right]
$$

where

$$
C(x):=\{y \in Z: \pi(y)=x\}, \quad x \in \mathbb{R}^{d} .
$$

Moreover, by (10.1) we have

$$
\hat{N} \cdot|C(x)|_{d} \leq 1, \quad x \in N
$$

Consider the point process $N^{\prime}:=\left\{x \in N: \hat{N} \cdot|C(x)|_{d}<1\right\}$. By (10.2) we have $Z=\mathbb{R}^{d}$ on the shift-invariant event $\left\{N^{\prime} \neq \varnothing\right\}$. Hence, (10.3) implies that

$$
\mathrm{P}_{N}^{*}\left(\hat{N} \cdot|C(0)|_{d}=1 \mid \mathcal{I}\right)=1 \quad \mathrm{P} \text {-a.s. on }\left\{N^{\prime} \neq \varnothing\right\} .
$$

On the other hand, by (10.4), on $\left\{N^{\prime}=\varnothing\right\}$ we almost surely have

$$
\begin{aligned}
\mathrm{P}_{N}^{*}\left(\hat{N} \cdot|C(0)|_{d}=1 \mid \mathcal{I}\right) & =\mathrm{E}\left[\hat{N}^{-1} \int_{[0,1]^{d}} \mathbf{1}\left(\hat{N} \cdot|C(x)|_{d}=1\right) N(\mathrm{~d} x) \mid \tau\right] \\
& =\mathrm{E}\left[\hat{N}^{-1} N\left([0,1]^{d}\right) \mid \mathcal{I}\right] \\
& =1 .
\end{aligned}
$$

Together with (10.5) this yields

$$
\mathrm{P}_{N}^{*}\left(\hat{N} \cdot|C(0)|_{d}=1\right)=1 .
$$

However, (10.3) then implies that $\mathrm{P}(0 \in Z)=1$, so the covariance of $Z$ gives $\mathrm{P}(x \in Z)=1$ for all $x \in \mathbb{R}^{d}$. By Fubini's theorem,

$$
\mathrm{E}\left[\left|\mathbb{R}^{d} \backslash Z\right|_{d}\right]=\mathrm{E}\left[\int \mathbf{1}(x \notin Z) \mathrm{d} x\right]=\int \mathrm{P}(x \notin Z) \mathrm{d} x=0 .
$$

Therefore, the cells $C^{\delta}(x):=\left\{y \in \mathbb{R}^{d}: \delta(y)=x\right\}, x \in N$, satisfy

$$
\left|C^{\delta}(x)\right|_{d}=|C(x)|_{d} \quad \mathrm{P} \text {-a.s., } \quad x \in N .
$$

Hence, (10.6) implies that $\delta$ is balanced. It follows from the first inequality in (10.1) that $\delta$ is P-proper. 


\section{Shift couplings}

Let $\mathrm{P}$ be a stationary probability measure on $(\Omega, \mathcal{A})$ and $N$ a flow-adapted point process such that $\mathrm{P}(0<\hat{N}<\infty)=1$. Theorems 10.1 and 9.1 provide a $\sigma(N)$-measurable random variable $\tau$ such that $N-\tau$ follows the modified Palm distribution of $N$, i.e.

$$
\mathrm{P}(N-\tau \in \cdot)=\mathrm{P}_{N}^{*}(N \in \cdot)
$$

The pair $(N, N-\tau)$ is called a shift coupling (see [13] and [14]), defined on the probability space supporting the stationary point process $N$. The remarkable fact is that the shift $\tau$ can be chosen to be a (deterministic) measurable function of $N$.

We may now ask whether there is random variable $\tau^{*}$ such that

$$
\mathrm{P}_{N}^{*}\left(N+\tau^{*} \in \cdot\right)=\mathrm{P}(N \in \cdot),
$$

and, thus, a shift coupling $\left(N, N+\tau^{*}\right)$, defined on the probability space $\left(\Omega, \mathcal{A}, \mathrm{P}_{N}^{*}\right)$. Owing to (5.7), general results of [13] show that this is possible on a suitable extension of $\left(\Omega, \mathcal{A}, \mathrm{P}_{N}^{*}\right)$. However, in contrast to (11.1), the next proposition shows that $\tau^{*}$ cannot be chosen to be a measurable function of $N$. In view of Proposition 4.4, this result is expected. In our proof we follow the authors of [3], who derived the result for the Palm distribution. To formulate the assumptions of the proposition we use the canonical framework of Example 2.1.

Proposition 11.1. Let $\mathrm{P}^{\prime}$ be a stationary probability measure on $(\boldsymbol{N}, \mathcal{N})$ such that the identity $N$ on $\mathrm{N}^{\mathrm{P}^{\prime}}$-a.s. has a positive, finite sample intensity. Let $\mathrm{P}^{*}$ be the modified Palm probability measure of $N$ defined with respect to $\mathrm{P}^{\prime}$. Let $N^{*}$ be a point process with distribution $\mathrm{P}^{*}$ and $\tau^{*}$ a random element in $\mathbb{R}^{d}$, both defined on the same probability space and such that $N^{*}+\tau^{*}$ has distribution $\mathrm{P}^{\prime}$. Then the conditional distribution of $\tau^{*}$ given $N^{*}$ is almost surely absolutely continuous. The density may be chosen to be smaller than $\tilde{N}$, where

$$
\tilde{N}=\liminf _{n \rightarrow \infty} \frac{N^{*}\left(B_{n}\right)}{\left|B_{n}\right|_{d}}
$$

and the $B_{n}$ are as in (5.2).

Proof. We assume that our probability space $(\Omega, \mathcal{A}, \mathrm{P})$ is given by $\Omega:=N \times(0,1)$, with $\mathcal{A}$ the product $\sigma$-field and $\mathrm{P}$ the product of $\mathrm{P}^{\prime}$ and the Lebesgue measure. For $(\varphi, t) \in \Omega$, we write $N(\omega):=\varphi$ and $\theta_{x} \omega:=(\varphi-x, t), x \in \mathbb{R}^{d}$. Then $\mathrm{P}$ is stationary and $N$ is adapted to the flow $\left\{\theta_{x}: x \in \mathbb{R}\right\}$. Using the conditional distribution of $\left(N^{*}, \tau^{*}\right)$ given $N^{*}+\tau^{*}$ and proceeding as in, e.g. Theorem 6.10 of [4], we can define a point process $N^{0}$ and a random variable $\tau$ on $(\Omega, \mathcal{A}, \mathrm{P})$ such that

$$
\left(N, N^{0}, \tau\right) \stackrel{\mathrm{D}}{=}\left(N^{*}+\tau^{*}, N^{*}, \tau^{*}\right)
$$

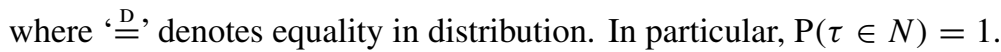

Now we choose a $\beta>1$ and a Borel set $B \subset \mathbb{R}^{d}$ and consider the measurable set

$$
A:=\left\{\varphi \in N: \mathrm{P}\left(\tau \in B \mid N^{0}=\varphi\right) \geq \beta h(\varphi)|B|_{d}\right\},
$$

where

$$
h(\varphi):=\liminf _{n \rightarrow \infty} \frac{\varphi\left(B_{n}\right)}{\left|B_{n}\right|_{d}}, \quad \varphi \in N
$$


Then

$$
\begin{aligned}
\beta|B|_{d} \mathrm{P}^{*}(A) & =\beta|B|_{d} \mathrm{P}\left(N^{0} \in A\right) \\
& \leq \mathrm{E}\left[h\left(N^{0}\right)^{-1} \mathbf{1}\left(N^{0} \in A\right) \mathrm{P}\left(\tau \in B \mid N^{0}\right)\right] \\
& =\mathrm{E}\left[h\left(N^{0}\right)^{-1} \mathbf{1}\left(N^{0} \in A\right) \mathbf{1}(\tau \in B)\right] \\
& =\mathrm{E}\left[h(N-\tau)^{-1} \mathbf{1}(N-\tau \in A) \mathbf{1}(\tau \in B)\right],
\end{aligned}
$$

where the last equality is due to

$$
(N-\tau, \tau) \stackrel{\mathrm{D}}{=}\left(N^{*}, \tau^{*}\right) \stackrel{\mathrm{D}}{=}\left(N^{0}, \tau\right) .
$$

Now we note that the sample intensity, $\hat{N}$, of $N$ P-a.s. equals $h(N-\tau)$. Indeed, it is sufficient to check this on $\{|\tau| \leq c\}$ for each $c>0$, which can be done using a standard argument from spatial ergodic theory (see, e.g. the proof of Corollary 10.19 of [4]). Hence,

$$
\begin{aligned}
\beta|B|_{d} \mathrm{P}^{*}(A) & \leq \mathrm{E}\left[\hat{N}^{-1} \mathbf{1}(N-\tau \in A) \mathbf{1}(\tau \in B)\right] \\
& \leq \mathrm{E}\left[\hat{N}^{-1} \int \mathbf{1}(N-x \in A) \mathbf{1}(x \in B) N(\mathrm{~d} x)\right] \\
& =|B|_{d} \mathrm{P}^{*}(A),
\end{aligned}
$$

where we have used the modified refined Campbell theorem, (5.6). Hence, if $0<|B|_{d}<\infty$ then

$$
\mathrm{P}\left(\tau \in B \mid N^{0}\right) \leq h\left(N^{0}\right)|B|_{d} \quad \mathrm{P} \text {-a.s. }
$$

Since $|B|_{d}=\lim _{n \rightarrow \infty}\left|G_{n}\right|_{d}$ for some decreasing sequence $G_{n} \supset B, n \in \mathbb{N}$, of open sets, (11.3) holds for any Borel set $B$. By taking $B$ from a countable algebra generating $\mathcal{B}^{d}$ and working with a regular version of $\mathrm{P}\left(\tau \in \cdot \mid N^{0}\right)$, we conclude that the exceptional set for (11.3) can be chosen independently of $B \in \mathscr{B}^{d}$. In view of (11.2), this implies both assertions of the proposition.

\section{Acknowledgements}

Large parts of this paper were written while the author held visiting positions at the Australian National University in Canberra and at the University of Melbourne in July 2005. The author is grateful for the hospitality shown to him and for the nice and stimulating atmosphere created by his hosts Professor D. Daley and Professor K. Borovkov. Thanks are also due to an anonymous referee, whose comments helped to improve the paper.

\section{References}

[1] Daley, D. J. And Vere-Jones, D. (1988). An Introduction to the Theory of Point Processes. Springer, New York.

[2] Heveling, M. and Last, G. (2005). Characterization of Palm measures via bijective point-shifts. Ann. Prob. 33, 1698-1715.

[3] Holroyd, A. E. And Peres, Y. (2005). Extra heads and invariant allocations. Ann. Prob. 33, 31-52.

[4] Kallenberg, O. (2002). Foundations of Modern Probability, 2nd edn. Springer, New York.

[5] Matthes, K., Kerstan, J. and Mecke, J. (1978). Infinitely Divisible Point Processes. John Wiley, Chichester.

[6] Mecke, J. (1967). Stationäre zufällige Masse auf lokalkompakten Abelschen Gruppen. Z. Wahrscheinlichkeitsth. 9, 36-58.

[7] Mecke, J. (1975). Invarianzeigenschaften allgemeiner Palmscher Maße. Math. Nachr. 65, 335-344.

[8] Neveu, J. (1977). Processus ponctuels. In École d'Été de Probabilités de Saint-Flour, VI (Lecture Notes Math. 598). Springer, Berlin, pp. 249-445. 
[9] NiEuwenhuis, G. (1989). Equivalence of functional limit theorems for stationary point processes and their Palm distributions. Prob. Theory Relat. Fields 81, 593-608.

[10] Nieuwenhuis, G. (1994). Bridging the gap between a stationary point process and its Palm distribution. Statist. Neerlandica 48, 37-62.

[11] SchneIder, R. And WeIL, W. (2000). Stochastische Geometrie. Teubner, Stuttgart.

[12] Stoyan, D., Kendall, W. S. And Mecke, J. (1995). Stochastic Geometry and Its Applications, 2nd edn. John Wiley, Chichester.

[13] Thorisson, H. (1996). Transforming random elements and shifting random fields. Ann. Prob. 24, $2057-2064$.

[14] Thorisson, H. (2000). Coupling, Stationarity, and Regeneration. Springer, New York. 